\begin{tabular}{ll} 
C O L L O Q U I U M & M A T H E M A T I C U M \\
\hline Vol. LXIV & 1993 \\
\hline
\end{tabular}

\title{
A CERTAIN PROPERTY OF ABELIAN GROUPS
}

BY

WITOLD SEREDYŃSKI AND JACEK ŚWIA̧TKOWSKI (WROCEAW)

\section{Statement of the result}

1.1. Definition. A subset $A$ of an abelian group $G$ is said to cover not less than $1 / n$ of the group if there exist $g_{1}, \ldots, g_{n} \in G$ such that $\bigcup_{i=1}^{n} g_{i} A=G$.

1.2. Remark. $g_{n}=e$ may be assumed in the above definition since $G=g_{n}^{-1} G=\bigcup_{i=1}^{n} g_{n}^{-1} g_{i} A$.

1.3. The Main Theorem. Let $G$ be an abelian group and $A_{1}, \ldots, A_{n}$ its disjoint subsets, each covering not less than $1 / n$ of the group. Then $\bigcup_{i=1}^{n} A_{i}=G$.

1.4. Remark. For obvious arithmetical reasons the theorem holds for all finite (not necessarily abelian) groups. On the other hand, it is easy to construct a counterexample in a free group with two generators. The question arises about the class of groups for which the theorem holds, but we do not give any ideas for the answer in this paper.

2. Reduction of the main theorem to a special case. In the following only abelian groups will be considered and therefore we will use the additive notation.

2.1. Lemma. Let $G$ be abelian and let sets $A_{i}$ cover not less than $1 / n$ of it. Assume that $A_{i} \cup \bigcup_{k=1}^{n-1}\left(g_{i k}+A_{i}\right)=G$ for $i=1, \ldots, n$ (compare 1.2). If Theorem 1.3 holds for the subgroup $H$ of $G$ generated by all elements $g_{i k}$ and for the sets $A_{i}^{\prime}=A_{i} \cap H$ then it holds for $G$.

Proof. The action of $H$ on its cosets in $G$ is the same as on itself, so the theorem holds for each coset, and consequently for the whole $G$.

2.2. Remark. Let $H$ be as in 2.1 and $A_{i}^{\prime}=A_{i} \cap H$. Let $Z^{n(n-1)}$ be the free abelian group with generators $e_{i k}, i=1, \ldots, n, k=1, \ldots, n-1$, and let $\phi: Z^{n(n-1)} \rightarrow H$ be a homomorphism such that $\phi\left(e_{i k}\right)=g_{i k}$. Put $B_{i}=$ $\phi^{-1}\left(A_{i}^{\prime}\right)$. Then $B_{i} \cup \bigcup_{k=1}^{n-1}\left(e_{i k}+B_{i}\right)=Z^{n(n-1)}$ for $i=1, \ldots, n$, thus each 
$B_{i}$ covers not less than $1 / n$ of $Z^{n(n-1)}$. If we prove that $\bigcup_{i=1}^{n} B_{i}=Z^{n(n-1)}$, then by surjectivity of $\phi$ we get $\bigcup_{i=1}^{n} A_{i}^{\prime}=H$.

According to 2.1 and 2.2, in order to prove the main theorem, it is enough to prove it in the following special case.

2.3. ThEOREM (The special case of the main theorem). Let $Z^{n(n-1)}$ be the free abelian group with generators $e_{i k}, i=1, \ldots, n, k=1, \ldots, n-1$, and let $B_{i}$ be its disjoint subsets with $B_{i} \cup \bigcup_{k=1}^{n-1}\left(e_{i k}+B_{i}\right)=Z^{n(n-1)}$ for $1, \ldots, n$. Then $\bigcup_{i=1}^{n} B_{i}=Z^{n(n-1)}$.

\section{Proof of the special case (Theorem 2.3)}

3.1. Let $Z_{i}^{n-1}$ be the subgroup of $Z^{n(n-1)}$ generated by $e_{i 1}, e_{i 2}, \ldots$ $\ldots, e_{i, n-1}$. Then $Z^{n(n-1)}=\bigoplus_{i=1}^{n} Z_{i}^{n-1}(\bigoplus$ denotes the free abelian product) and therefore we may identify $Z^{n(n-1)}$ with the cartesian product $Z_{1}^{n-1} \times Z_{2}^{n-1} \times \ldots \times Z_{n}^{n-1}$.

3.2. Definition. For $a \in Z^{n(n-1)}$ let $K_{a}^{i}=\left\{a, a-e_{i 1}, a-e_{i 2}, \ldots, a-\right.$ $\left.e_{i, n-1}\right\}$.

3.3. Remark. Note that for each $a \in Z^{n(n-1)}$ we can find in $K_{a}^{i}$ an element which belongs to $B_{i}$. If it is not $a$ then, since $B_{i} \cup \bigcup_{k=1}^{n-1}\left(e_{i k}+B_{i}\right)=$ $Z^{n(n-1)}, a \in e_{i k}+B_{i}$ for some $k$, and then $a-e_{i k} \in B_{i}$.

3.4. Definition. Let $a \in Z^{n(n-1)}$ and let $a=a_{1}+\ldots+a_{n}$ be its expansion with respect to the free abelian product of 3.1, i.e. $a_{i} \in Z_{i}^{n-i}$. Then define $K_{a}=K_{a_{1}}^{1} \times \ldots \times K_{a_{n}}^{n}$.

3.5. Lemma. For each $i \in\{1, \ldots, n\}$ and each $a \in Z^{n(n-1)}, K_{a}$ is a disjoint sum of $n^{n-1}$ sets of the form $K_{x}^{i}$.

Proof. Assume $i=n$. Then

$$
K_{a}=\left(K_{a_{1}}^{1} \times \ldots \times K_{a_{n-1}}^{n-1}\right) \times K_{a_{n}}^{n}=\underset{y \in K_{a_{1}}^{1} \times \ldots \times K_{a_{n-1}}^{n-1}}{\bigcup}\{y\} \times K_{a_{n}}^{n},
$$

which is a disjoint sum of sets of the desired form. For other $i$ the proof is the same.

3.6. Remark. According to 3.3 the sets $K_{a}$ and $B_{i}$ have at least $n^{n-1}$ elements in common. Since $B_{i}$ are disjoint an arithmetical argument proves that $K_{a} \subset \bigcup_{i=1}^{n} B_{i}$.

3.7. LEMMA. $Z^{n(n-1)}$ is a disjoint sum of sets of the form $K_{a}$.

Proof. It will be proved in the next section that $Z_{i}^{n-1}$ is a disjoint sum of sets of the form $K_{x}^{i}$, i.e. $Z^{n-1}=\bigcup_{x \in I_{i}} K_{x}^{i}$ for each $i$ and some $I_{i} \subset Z_{i}^{n-1}$. 
Thus

and the lemma follows.

$$
Z^{n(n-1)}=\bigcup_{\left(x_{1}, \ldots, x_{n}\right) \in I_{1} \times \ldots \times I_{n}} K_{x_{1}}^{1} \times \ldots \times K_{x_{n}}^{n}
$$

Theorem 2.3 now follows from 3.6 and 3.7.

\section{Combinatorial fact used in the proof of Lemma 3.7}

4.1. Proposition (used in 3.7). Let $Z^{n-1}$ be a free abelian group with generators $e_{1}, \ldots, e_{n-1}$. For $x \in Z^{n-1}$ put $K_{x}=\left\{x, x-e_{1} \ldots, x-e_{n-1}\right\}$. Then $Z^{n-1}$ is a disjoint sum of sets of the form $K_{x}$.

Before proving the proposition we give some notations and lemmas.

4.2. Notation. Let $Z_{n}$ be the group of integers $(\bmod n)$, i.e. a cyclic group of $n$ elements. Denote by $a_{i}, i \in\{0,1, \ldots, n-1\}$, the generators in $\left(Z_{n}\right)^{n-1}$ of the form $(1,0, \ldots, 0),(0,1,0, \ldots, 0), \ldots,(0, \ldots, 0,1)$. Put $x_{i}=$ $a_{i}+i a_{n-1}$ for $i=1, \ldots, n-2$, and denote by $V$ the subgroup of $\left(Z_{n}\right)^{n-1}$ generated by $x_{1}, \ldots, x_{n-2}$.

4.3. LEMMA. The elements $x_{1}, \ldots, x_{n-2}$ are free abelian generators of $V$ over $Z_{n}$

Proof. Suppose $\sum_{i=1}^{n-2} k_{i} x_{i}=0,0 \leq k_{i}<n$. Then $\sum_{i=1}^{n-2} k_{i}\left(a_{i}+\right.$ $\left.i a_{n-1}\right)=\sum_{i=1}^{n-2} k_{i} a_{i}+\left(\sum_{i=1}^{n-2} i k_{i}\right) a_{n-1}=0$, which implies $k_{i}=0$ for $i=$ $1, \ldots, n-2$ and the lemma follows.

4.4. R e mark. Lemma 4.3 implies that $V$ consists of $n^{n-2}$ elements.

4.5. Lemma. For $1 \leq i \leq n-1, a_{i} \notin V$.

Proof. Suppose $a_{i}=\sum_{l=1}^{n-2} k_{l} x_{l}=\sum_{l=1}^{n-2} k_{l} a_{l}+\left(\sum_{l=1}^{n-2} l k_{l}\right) a_{n-1}$. If $i \neq n-1$ then $k_{i}=1$ and $k_{l}=0$ for $l \neq i$, but then $\sum_{l=1}^{n-2} l k_{l}=i \neq 0$ $(\bmod n)$, a contradiction.

If $i=n-1$ then $k_{l}=0$ for each $l$, which contradicts $\sum_{l=1}^{n-2} l k_{l}=1$ $(\bmod n)$.

4.6. Lemma. For $1 \leq i, j \leq n, i \neq j, a_{i}-a_{j} \notin V$.

Proof. Case 1: $i \neq j, i \neq n-1$ and $j \neq n-1$. Suppose $a_{i}-a_{j}=$ $\sum_{l=1}^{n-2} k_{l} x_{l}=\sum_{l=1}^{n-2} k_{l} a_{l}+\left(\sum_{l=1}^{n-2} l k_{l}\right) a_{n-1}$. Then $k_{i}=1, k_{j}=-1(\bmod n)$, $k_{l}=0$ for $l \neq i, j$ and consequently $\sum_{l=1}^{n-2} l k_{l}=i-j \neq 0(\bmod n)$, a contradiction.

Case 2: $i \neq j, i=n-1$. By the argument of the previous case we get $k_{j}=-1(\bmod n), k_{l}=0$ for $l \neq j$, and consequently $\sum_{l=1}^{n-2} l k_{l}=-j$ $(\bmod n)$, which contradicts $\sum_{l=1}^{n-2} l k_{l}=1(\bmod n)$ since $1 \leq j<n-1$. This completes the proof. 
4.7. Lemma. For $x \in\left(Z_{n}\right)^{n-1}$ define $K_{x}=\left\{x, x-a_{1}, \ldots, x-a_{n-1}\right\}$. Then $\left\{K_{x}: x \in V\right\}$ is a disjoint family and covers $\left(Z_{n}\right)^{n-1}$.

Proof. Suppose that $K_{x_{1}} \cap K_{x_{2}} \neq \emptyset$ for some $x_{1}, x_{2} \in V, x_{1} \neq x_{2}$. Then either $x_{1}=x_{2}-a_{i}, x_{2}=x_{1}-a_{i}$ or $x_{1}-a_{i}=x_{2}-a_{j}$ for some $i, j \in\{1, \ldots, n-1\}$. The first two cases imply $a_{i} \in V$ and the third implies $i \neq j$ (since $\left.x_{1} \neq x_{2}\right)$ and $a_{i}-a_{j} \in V$, but none of these is possible according to 4.5 and 4.6. It follows from 4.4 that $\bigcup\left\{K_{x}: x \in V\right\}$ consists of $n^{n-1}$ elements and thus equals $\left(Z_{n}\right)^{n-1}$.

4.8. Pro of of 4.1. Let $\phi: Z^{n-1} \rightarrow\left(Z_{n}\right)^{n-1}$ be the canonical homomorphism satisfying $\phi\left(e_{i}\right)=a_{i}$, and $V^{\prime}=\phi^{-1}(V)$. Then from 4.7 it follows that the family $\left\{K_{x}: x \in V^{\prime}\right\}$ is a disjoint cover of $Z^{n-1}$, which completes the proof.

Witold Seredyński

INSTITUTE OF MATHEMATICS TECHNICAL UNIVERSITY OF WROCEAW

WYBRZEŻE WYSPIAŃSKIEGO 27

50-370 WROCEAW, POLAND
Jacek Świątkowski

INSTITUTE OF MATHEMATICS WROCEAW UNIVERSITY PL. GRUNWALDZKI $2 / 4$ 50-384 WROCŁAW, POLAND 